\title{
INTERVENÇÕES FAMILIARES NO PRIMEIRO EPISÓDIO PSICÓTICO: FACILITADORES E BARREIRAS NA PERSPECTIVA DA EQUIPE DE SAÚDE
}

\section{FAMILY INTERVENTIONS IN THE FIRST-EPISODE PSYCHOSIS: FACILITATORS AND BARRIERS FROM THE PERSPECTIVE OF THE HEALTH TEAM}

\section{INTERVENCIONES FAMILIARES EN EL PRIMER EPISODIO PSICÓTICO: FACILITADORES Y BARRERAS EN LA PERSPECTIVA DEL EQUIPO DE SALUD}

\author{
Lorena Fendrich $^{1}$, Heloisa França Badagnan ${ }^{2}$, Luiza Elena Casaburi ${ }^{3}$, Ana Carolina \\ Guidorizzi Zanetti ${ }^{4}$, Sueli Aparecida Frari Galera ${ }^{5}$
}

\section{RESUMO}

Objetivo: Identificar e analisar as possibilidades e barreiras percebidas por profissionais de saúde para implantar e manter, na rotina de um ambulatório de primeiro episódio psicótico, o cuidado às famílias de portadores de transtorno mental. Método: Estudo qualitativo, cuja coleta de dados foi realizada através de duas sessões de grupo focal com a equipe de um ambulatório de primeiro episódio psicótico. Resultados: Os profissionais participantes ressaltaram a importância da família no início do tratamento, e suas necessidades. Essa percepção foi interpretada como um facilitador para a inclusão da família. As barreiras para uma participação mais ativa identificadas nas discussões estão relacionadas à própria família, à estrutura e organização do serviço, e aos profissionais. A supervalorização do modelo biomédico está presente em todas as barreiras. Conclusão: O estudo reforçou a importância da inclusão da família no tratamento desses indivíduos, e contribui apontando os facilitadores e barreiras para efetivar esta proposta.

Descritores: Enfermagem Psiquiátrica; Serviços de Saúde Mental; Terapia Familiar; Prática Profissional; Transtornos Psicóticos.

\footnotetext{
1 Doutoranda do Programa de Enfermagem Psiquiátrica da Escola de Enfermagem de Ribeirão Preto.

2 Escola de Enfermagem de Ribeirão Preto da Universidade de São Paulo - EERP/USP

3 Universidade de Uberaba (UNIUBE)

4 Escola de Enfermagem de Ribeirão Preto da Universidade de São Paulo - EERP/USP

5 Escola de Enfermagem de Ribeirão Preto da Universidade de São Paulo - EERP/USP
} 


\section{ABSTRACT}

Aim: Identify and analyze the possibilities and barriers perceived by health professionals to deploy and maintain, in a routine of an outpatient first episode psychosis service, care for the families of patients with mental disorders. Method: Qualitative study. For data collection, were realized two sessions of a focus group with the team of a first episode psychosis outpatient service. Results: Professional participants stressed the importance of the family at the beginning of treatment and their needs. This perception was interpreted as facilitators to the inclusion of the family. The barriers to a more active participation identified in the discussions are related to the family, the structure and organization of the service, and the professionals. The overvaluation of the biomedical model is present in all barriers. Conclusion: The study reinforced our understanding of the importance of including the family in the care, and contributes pointing the facilitators and barriers to effect this proposal.

Descriptors: Psychiatric Nursing; Mental Health Services; Family Therapy; Professional Practice; Psychotic disorders.

\section{RESUMEN}

Objetivo: Identificar y analizar las comodidades y barreras percibidas por los profesionales de la salud a implementar y mantener, en la rutina de un ambulatorio de primer episodio psicótico, el cuidado de los familiares de pacientes con trastornos mentales. Método: Estudio cualitativo. Para la recolección de datos, se realizaron dos sesiones de grupos focales con el equipo de una clínica para el primer episodio psicótico. Resultados: Profesionales participantes destacaron la importancia de la familia en el inicio del tratamiento, y sus necesidades. Esta percepción fue interpretada como un facilitador para la inclusión de la família. Las barreras para una participación más activa identificadas en las discusiones están relacionados con la propia familia, a la estructura y organización del servicio, ya los profesionales. La sobrevaloración del modelo biomédico está presente en todas estas barreras. Conclusión: El estudio refuerza la importancia de la inclusión de la familia en el tratamiento, y contribuye señalando los facilitadores y los obstáculos a la realización de esta propuesta.

Descriptores: Enfermería Psiquiátrica; Servicios de Salud Mental; Terapia Familiar; Practica Profesional; Los Trastornos Psicóticos.

\section{INTRODUÇÃO}

A psicose, caracterizada pela presença de delírios e alucinações, pode ocorrer em vários transtornos mentais, sendo a esquizofrenia e o transtorno afetivo bipolar os mais conhecidos. O primeiro episódio psicótico (PEP) normalmente ocorre entre a adolescência e início da vida adulta. ${ }^{1}$ Esse início do 
adoecimento mental causa impacto profundo na vida do indivíduo e de sua família. Essa experiência tem sido comparada ao trauma vivido por vítimas de catástrofes. Os membros da família ficam confusos, se esforçando para entender as mudanças de comportamento de seu ente, e são comuns emoções como angústia, ansiedade e culpa. Neste sentido, é importante acolher a família desde o início do adoecimento. ${ }^{2}$

A literatura mostra que as famílias estão sofrendo com experiências emocionais e práticas difíceis pois, muitas vezes despreparadas, se deparam com desafios ao assumirem o papel de cuidadoras. ${ }^{3}$ As estratégias usadas para lidar com os problemas relacionados aos cuidados e ao adoecimento são frequentemente insuficientes. Nesse sentido, estudos destacam a necessidade de oferecer intervenções familiares eficientes para melhorar o estado de saúde mental dos cuidadores e potencializar seu papel na recuperação do adoecido. ${ }^{3,4}$

Alguns trabalhos sugerem que, ao compartilharem encargos e oferecerem apoio, as intervenções familiares podem minimizar os efeitos dos eventos estressores, promover melhora do quadro clínico do adoecido, diminuir as recaídas e o número de internações psiquiátricas. Além disso, as famílias relatam frequentemente a necessidade de informação e apoio profissional para ajudálas a lidar com a doença de um de seus membros. ${ }^{3,5,6}$

Estudo qualitativo conduzido em um hospital universitário na Noruega para explorar os benefícios da intervenção familiar percebidos por indivíduos que tiveram um primeiro episódio psicótico e seus familiares, apontou que houve um maior desenvolvimento de insight $\mathrm{e}$ aceitação da doença, melhoria nas habilidades de comunicação, planejamento e resolução de problemas. ${ }^{7}$

$\mathrm{Na}$ Austrália, um estudo qualitativo realizado em um serviço de saúde mental avaliou uma intervenção destinada aos familiares cuidadores de indivíduos que vivenciaram um primeiro episódio psicótico. Seus achados evidenciaram que a participação das famílias nesse tipo de atividade contribuiu para reduzir seu sentimento de isolamento, seu estigma, fez com que seus membros se sentissem ouvidos, aumentou seu conhecimento sobre a doença mental e aprimorou suas habilidades para apoiar o familiar adoecido. $^{8}$ 
Embora os estudos apresentados acima indiquem que a intervenção familiar tem resultados positivos, a inclusão da família no início do adoecimento e início do tratamento ainda não é procedimento de rotina em muitos serviços. ${ }^{9} \mathrm{E}$, mesmo quando faz parte da rotina os familiares ainda não se sentem totalmente incluídos no processo de tratamento. O que impede ou inibe a prática da intervenção familiar nos serviços de saúde mental? Frente a essa questão, o presente estudo teve como objetivo identificar e analisar os facilitadores e barreiras percebidos por profissionais de saúde para implantar e manter, na rotina de um ambulatório de primeiro episódio psicótico, o cuidado às famílias de portadores de transtorno mental.

\section{MÉTODO}

Trata-se de um estudo qualitativo que coletou dados por meio de grupo focal com os profissionais que compõem a equipe multidisciplinar de um Ambulatório de Primeiro Episódio Psicótico (APEP) de um hospital escola.

O APEP oferece tratamento medicamentoso e psicossocial por meio do grupo de psicoeducação para familiares e adoecidos e atendimento familiar individual com base no Modelo Calgary de Avaliação e de Intervenção na Família. ${ }^{10}$ Por se tratar de instituição de ensino, a equipe multidisciplinar do APEP é dividida em equipe fixa e equipe flutuante. A equipe fixa é composta por dois médicos psiquiatras supervisores, uma psicóloga, e duas enfermeiras especialistas responsáveis pelo atendimento individual das famílias, totalizando cinco profissionais. Já a equipe flutuante é composta por nove profissionais, sendo cinco médicos do segundo ano de residência em psiquiatria, que realizam os atendimentos médicos individuais, dois residentes do terceiro ano e duas enfermeiras pós-graduandas, que realizam estágio no APEP com o intuito de praticar atividades com os familiares.

Todos os profissionais foram convidados a participar. O convite foi feito pessoalmente e por e-mail por uma das pesquisadoras. Participaram do estudo sete profissionais, sendo todos da equipe fixa, e dois da equipe flutuante (uma médica residente e uma enfermeira pósgraduanda). Os outros profissionais não participaram devido à incompatibilidade de horários.

Ressaltamos que o atendimento individual das famílias faz parte de projeto de extensão de duas pesquisadoras deste 
trabalho, estando os autores desta pesquisa próximos da equipe do APEP. Esta condição pode configurar um viés importante que pode limitar o estudo. No entanto, acreditamos que esta condição não afeta a relevância do trabalho, pois existem poucos serviços como o APEP no Brasil. Além disso, nosso referencial mostra que um elo colaborativo entre pesquisadores e profissionais da assistência permite benefícios na prestação do cuidado tanto a níveis individuais quanto coletivos, bem como defende o grande potencial das abordagens participativas na transferência de conhecimento para a efetiva transformação da prática. ${ }^{10}$ Para garantir a qualidade dos dados, estes foram amplamente discutidos com a equipe.

A coleta de dados foi realizada entre os meses de setembro a dezembro de 2014 por meio de duas sessões de grupo focal, realizadas em local reservado, gravadas em áudio, e contou com uma pesquisadora como coordenadora do grupo e outra como observadora, que registrava anotações em um diário de campo. $\mathrm{Na}$ primeira sessão, foi utilizado um roteiro com as seguintes questões sobre a importância e os meios de inclusão da família no cuidado de pessoas no primeiro episódio psicótico: “Como vocês entendem o papel da família no cuidado ao paciente? No serviço onde vocês trabalham, é realizada alguma forma de interação com os familiares? Quais as dificuldades percebidas por vocês para incluir a família no cuidado aos pacientes deste serviço? E quais as facilidades?”. Após a realização dessa sessão, que durou 30 minutos, foi realizada a transcrição da discussão por uma das pesquisadoras, e sua leitura. Em seguida, foi construída uma narrativa sobre os temas discutidos, a qual foi apresentada junto à transcrição aos participantes no segundo grupo focal, com o intuito de validação das informações obtidas e aprofundamento dos temas identificados. $\mathrm{O}$ segundo grupo focal durou 59 minutos.

A análise do material obtido com a realização dos grupos focais consistiu em um primeiro momento na transcrição da discussão gravada na íntegra, com identificação dos participantes, e leitura compreensiva do material. Em uma segunda etapa, houve exploração desse material e a partir da transcrição foi elaborada a narrativa do grupo focal. Essa construção narrativa consistiu fundamentalmente em uma estratégia de tornar denso o material, mantendo-se fiel à história que nele se conta, mas não necessariamente em sua sequência 
temporal e tampouco reproduzindo as formas lexicais do grupo. ${ }^{11}$ Para tal, duas pesquisadoras realizaram uma primeira operação de reconhecimento de núcleos argumentais. Em seguida elaboraram a narrativa do grupo, que foi apresentada para leitura aos participantes na segunda sessão de grupo focal. Nesta sessão os participantes aprofundaram as discussões sobre os temas apresentados e colaboraram na redação final da narrativa.

O segundo encontro de grupo focal contou com a participação de um médico psiquiatra da equipe fixa, uma psicóloga, duas enfermeiras especialistas, uma enfermeira pós-graduanda, mais a coordenadora e a observadora.

O projeto da pesquisa foi aprovado pelo Comitê de Ética da Escola de Enfermagem de Ribeirão Preto, mediante protocolo CAAE 33677114.5.0000.5393. Os participantes foram esclarecidos e assinaram o Termo de Consentimento Livre e Esclarecido.

\section{RESULTADOS}

A partir da análise das discussões dos grupos focais, elaboramos duas categorias: facilitadores e barreiras. A primeira, trata das possibilidades que favorecem a inclusão da família no atendimento oferecido pelo APEP. Foram identificados dois tipos de facilitadores, como a compreensão do papel do familiar pelos profissionais do serviço, e a oferta de intervenções familiares no primeiro episódio psicótico. A segunda categoria trata das barreiras para a efetivação da intervenção familiar. Foram identificados três tipos de barreiras, relacionadas à própria família, aos profissionais e ao serviço.

\section{A compreensão sobre o papel da}

\section{família}

Os participantes do estudo afirmaram que a família é muito importante $e$ essencial (psiquiatra I) no atendimento de pessoas no primeiro episódio psicótico. Afirmaram que com o surgimento do surto psicótico o paciente perde momentaneamente a autonomia e precisa de alguém que se responsabilize por seu cuidado (residente). Outro argumento que valoriza o papel de cuidador da família é a própria política de saúde adotada em nosso país. De acordo com o que preconiza a Reforma Psiquiátrica brasileira, é a família quem irá assumir o cuidado em casa, ministrar medicações, dar amparo, observar o comportamento (psiquiatra II). 


\section{A oferta de intervenções}

\section{familiares no primeiro episódio psicótico}

Por outro lado, a família está impactada pelo surgimento da psicose, necessitando de apoio e informação para assumir o papel de cuidadora, principalmente no primeiro episódio psicótico (enfermeira pósgraduanda), como é o caso dos indivíduos em tratamento no serviço estudado.

Os profissionais salientaram que apesar de não ser uma exigência do serviço, os familiares geralmente comparecem aos retornos devido ao estado do familiar adoecido. Os pacientes atendidos geralmente se encontram em um estado muito grave, exigindo assim que uma pessoa os acompanhe nos retornos (psicóloga).

$\mathrm{Na}$ opinião dos profissionais, a presença da família nos atendimentos é um momento importante para a equipe incentivá-la a participar das atividades oferecidas pelo ambulatório, isto é, do grupo de familiares e do atendimento individual da família: os familiares têm que esperar a consulta médica e podem aproveitar este momento participando do grupo de psicoeducação para familiares e pacientes (psiquiatra II).

Outro aspecto que incentiva a participação dos adoecidos e seus familiares no grupo é a existência de uma equipe fixa que tem como responsabilidade conduzir os atendimentos à família, criando um vínculo com as famílias acompanhadas (enfermeira especialista I). Além disso, estas atividades representam um espaço para ser ouvido, tirarem dúvidas e no caso do grupo, trocarem experiências com outros indivíduos que enfrentam situação semelhante (residente).

Embora o ambulatório de primeiro episódio psicótico tenha a proposta de atender as famílias e sua equipe esteja motivada para isso, constituindo assim um facilitador para a inclusão da família na assistência, todos os profissionais concordaram que os familiares participam pouco do grupo de psicoeducação e dos atendimentos individuais para a família. A discussão sobre os fatores que podem atuar como obstáculos para a participação familiar apontou três barreiras importantes, citadas a seguir.

\section{Barreiras relacionadas à própria}

\section{família}

Os profissionais apontaram a própria família como sendo uma barreira importante para a pouca participação nos grupos e nos atendimentos individuais, embora haja um incentivo por parte da equipe convidando-a para as atividades. Os profissionais argumentaram que o aspecto financeiro pode dificultar a participação dos familiares, pois grande parte dos 
utentes depende de ônibus para ir ao serviço de saúde e muitas vezes a família não tem condições de pagar a passagem (psiquiatra II). Outro aspecto é a restrição de horário, pois familiares que trabalham fora geralmente precisam retornar ao trabalho o mais rápido possível.

Há também a questão da dificuldade de compreensão por parte do adoecido e do familiar sobre os benefícios das intervenções. Muitas vezes, apesar de todo trabalho realizado para explicar, eles não reconhecem a importância de participarem. Para alguns profissionais esta dificuldade está relacionada à nossa cultura, pois existe uma supervalorização do modelo biomédico - intervenção medicamentosa (residente) em detrimento do modelo psicossocial.

\section{Barreiras relacionadas ao Serviço}

No início, o serviço preconizava a realização de dois grupos de psicoeducação: um para pacientes, e outro para familiares (psiquiatra II). No entanto, a falta de salas e de profissionais disponíveis para realizar os dois atendimentos simultaneamente fez com que a equipe decidisse realizar um único grupo, para adoecidos e familiares juntos. Destaca-se que essa configuração também funciona como uma barreira, na medida em que familiares e adoecidos podem não se sentir à vontade para colocar as questões que gostariam de discutir.

Outro aspecto da organização do serviço apontado como barreira é o horário do grupo, que é oferecido concomitante aos atendimentos médicos. Como a demanda do serviço é grande, quando um número elevado de pacientes participa do grupo, as consultas médicas acabam atrasando e se tornam mais corridas. Isso gera uma resistência tanto dos pacientes, que optam por não participar do grupo e serem atendidos pelo médico mais cedo e de forma mais tranquila; quanto dos médicos, que não querem o acúmulo de consultas para o horário depois do grupo (psiquiatra I).

\section{Barreiras relacionadas aos}

\section{Profissionais}

A inclusão da família no atendimento ao portador de transtorno mental ainda é recente em nossa conjuntura, e os profissionais nem sempre estão preparados para realizá-la. No contexto do ambulatório, o aprendizado de maneira sistematizada e com embasamento teórico sobre intervenção familiar está direcionado ao residente do terceiro ano. No entanto, quem realiza o atendimento médico são os residentes do segundo ano, que ainda não tiveram um maior contato com as intervenções familiares. 
Dessa forma, alguns médicos do segundo ano de residência podem não acreditar que as intervenções junto às famílias possam trazer algum tipo de benefício para o portador de transtorno mental, para a família ou para o serviço. Isso pode se tornar um empecilho para o incentivo da participação das famílias nas atividades de intervenção, tanto individual quanto grupal, pois eles ainda não passaram pela experiência e talvez eles tenham dificuldade de se apropriar dessa intervenção para convencer o paciente (psiquiatra II).

\section{DISCUSSÃO}

Este estudo identificou que desde a sua concepção o APEP tem a proposta de atender os familiares dos portadores de transtorno mental, pois se entende que o surgimento da psicose causa um forte impacto na família e que é necessário ajudá-la na compreensão do adoecimento. Além disso, no início do tratamento os adoecidos ainda se encontram muito sintomáticos e estão momentaneamente dependentes de cuidados. Essa percepção é um elemento facilitador da inclusão da família no planejamento dos cuidados ao portador de transtorno mental. Nesse sentido, o APEP preconiza uma prática baseada em evidências, pois a literatura aponta que é fundamental incluir a família precocemente no plano de tratamento. ${ }^{12}$

A intervenção precoce junto à família no processo de tratamento e recuperação dos adoecidos, por meio do oferecimento de apoio e de informações, promove com êxito uma maior participação dos cuidadores no tratamento de seus familiares. Além disso, melhora a adesão à medicação, reduz as taxas de recaída, melhora o resultado funcional e a qualidade de vida dos adoecidos. ${ }^{1,13} \mathrm{Em}$ relação à família, aumenta o conhecimento do grupo, promove sua saúde mental e fortalece suas estratégias de enfrentamento. ${ }^{14}$

Os profissionais participantes de nosso estudo ressaltaram a importância da família no início do tratamento, e também relataram suas necessidades nesse momento. Porém, reconheceram que os adoecidos e seus familiares participam pouco das atividades oferecidas a eles. As barreiras para uma participação mais ativa identificadas nas discussões estão relacionadas à própria família, ao serviço, e aos profissionais.

Dentre os motivos que influenciam negativamente a participação das famílias nas atividades de intervenção, foram 
citados a crença no modelo biomédico como o único eficaz, além de questões pessoais, como ter horário restrito, dificuldade de transporte, e dificuldades financeiras.

A respeito do modelo biomédico, também identificado como modelo médico-assistencial privatista, médico hegemônico, ou hospitalocêntrico, podemos afirmar que este predomina no sistema de saúde brasileiro e nos últimos anos se consolidou como o paradigma dominante no campo da saúde mental. ${ }^{15}$ Sua concepção de prática é pautada no diagnóstico e na terapêutica medicamentosa, e está centrado na figura do médico. ${ }^{16}$ Por isso, há uma tendência a se optar apenas pelo tratamento medicamentoso, desvalorizando em contrapartida outras formas de assistência.

Outro fator que representa uma importante barreira à implementação de práticas baseadas em evidências em serviços de saúde mental é o tempo. Estudo cujo objetivo foi identificar barreiras para implementação de psicoeducação familiar descreveu que demandas conflitantes de tempo são comuns já que os membros da família geralmente enfrentam encargos significativos. Tais encargos podem representar obstáculos ao comparecimento e permanência às sessões de psicoeducação familiar. $^{17}$

Sobre a questão financeira, estudo realizado no Brasil com familiares cuidadores, argumenta que a presença do transtorno mental na família implica que o adoecido deixe de trabalhar por um período e, muitas vezes, outro familiar altere sua situação de trabalho em função da demanda da atividade de cuidar do parente adoecido. ${ }^{6}$

Em relação às barreiras impostas pelo serviço, verificamos nos resultados que embora este preconize as atividades de intervenção familiar, ele não dispõe de estrutura adequada para isso. A literatura também aponta essa falta de uma estrutura apropriada nos serviços de saúde para a realização do trabalho com famílias. ${ }^{18}$ Aponta ainda que a ausência de uma cultura organizacional envolvendo $\mathrm{o}$ trabalho com famílias pode ser devido à influência de paradigmas tradicionais baseados na predominância de modelos biológicos da doença mental, que tendem a minimizar o foco no contexto social do indivíduo. Nesse sentido, há a necessidade de uma mudança no contexto 
organizacional dos serviços para que a participação familiar no tratamento dos adoecidos possa ser implementada de forma eficaz. ${ }^{19}$

Outra dificuldade identificada para implantar as atividades de intervenção familiar diz respeito à falta de conhecimento e de envolvimento de alguns profissionais do serviço. Os participantes explicaram que os médicos residentes que realizam a consulta médica, ainda não passaram por estágio onde a família é valorizada e a intervenção familiar é ensinada.

Estudo desenvolvido nos Estados Unidos descreve que a falta de experiência, motivação, e/ou interesse dos profissionais sobre a adoção e implementação de práticas baseadas em evidências na rotina de uma instituição podem acarretar o não envolvimento destes para com as atividades voltadas para a família. ${ }^{20}$ Além disso, observamos em nossos resultados que a supervalorização do modelo biomédico também ocorre entre os profissionais. As conclusões dos participantes desta pesquisa coincidem com as de outros autores quando afirmam que muitas vezes há uma supervalorização do modelo biomédico por parte de alguns profissionais que, desta forma, enxergam as intervenções psicossociais e as interações com as famílias como atividades supérfluas, e não acreditam em sua eficácia. ${ }^{19}$

Verificamos neste estudo que a supervalorização do modelo biomédico está presente em todas as barreiras: as relacionadas à família, ao serviço, e aos profissionais. Um dos fatores que dificultam vencer esta crença é a falta de preparo dos profissionais. A literatura sugere como alternativa para driblar esses problemas relativos à equipe $\mathrm{o}$ oferecimento de suporte e treinamento aos profissionais que atuam na saúde mental para realizarem trabalhos baseados em evidências que incluam as famílias. ${ }^{20} \mathrm{O}$ objetivo final é implementar efetivamente as intervenções familiares na rotina de um serviço. Todos os membros da equipe devem ser abordados e regularmente treinados e supervisionados. $\mathrm{O}$ desenvolvimento de uma estrutura sólida para as intervenções pode inclusive ser favorável ao envolvimento das famílias, desde que sejam asseguradas suas necessidades individuais. 


\section{CONCLUSÃO}

O objetivo desta pesquisa foi identificar e analisar os facilitadores e as barreiras para implantar as intervenções familiares na rotina de um serviço de saúde mental. Como facilitadores, identificou-se que os profissionais reconhecem $\mathrm{o}$ sofrimento da família e suas necessidades de informação e acolhimento. Estão, portanto, motivados para interagir com as famílias dos adoecidos. As barreiras aqui identificadas estão relacionadas à família, à organização do serviço, e aos profissionais, e são semelhantes aos achados de outros estudos nacionais e internacionais.

Concluímos que o estudo reforçou nossa compreensão sobre a importância da inclusão da família no atendimento de pessoas no primeiro episódio psicótico. Contribuiu apontando os facilitadores e as barreiras para efetivar essa proposta, e apontou a necessidade de incorporar conhecimentos, bem como o desenvolvimento de habilidades para o trabalho com famílias.

A principal limitação do estudo é que as informações por ele obtidas consistem somente da perspectiva dos profissionais que atuam no serviço. A literatura aponta que além dos profissionais, é importante incluir o adoecido, a família, e também os profissionais ligados à tomada de decisão na gestão dos serviços.

\section{REFERÊNCIAS}

1. Lutgens D, Iyer S, Joober R, Brown TG, Norman R, Latimer E, et al. A five-year randomized parallel and blinded clinical trial of an extended specialized early intervention vs. regular care in the early phase of psychotic disorders: study protocol. BMC Psychiatry. 2015; 15:22.

2. Leggatt M, Woodhead G. Family peer support work in an early intervention youth mental health service. Early Interv Psychiatry. 2016; 10(5):446-51.

3. Caqueo-Urízar A, Rus-Calafell M, Craig TK, Irarrazaval M, Urzúa A, Boyer L, et al. Schizophrenia: Impact on Family Dynamics. Curr Psychiatry Rep. 2017; 19(1):2.

4. Reinares M, Bonnín CM, HidalgoMazzei D, Sánchez-Moreno J, Colom F, Vieta E. The role of family interventions in bipolar disorder: a systematic review. Clin Psychol Rev. 2016; 43:47-57.

5. Hsiao CY, Yun-Fang T. Factors associated with the perception of family nursing practice among mental health nurses in Taiwan. $\mathbf{J}$ Fam Nurs. 2015; 21(4):508-28.

6. Bessa JB, Waidman MAP. Família da pessoa com transtorno mental e suas necessidades na assistência psiquiátrica. Texto \& contexto Enferm. 2013; 22(1):61-70. 
7. Nilsen L, Frich JC, Friis S, Røssberg JI. Patients' and family members' experiences of a psychoeducational family intervention after a first episode psychosis: a qualitative study. Issues Ment Health Nurs. 2014; 35 (1):58-68.

8. Petrakis M, Bloom H, Oxley J. Family perceptions of benefits and barriers to first episode psychosis carer group participation. Soc Work Ment Health. 2014; 12(2):99-116.

9. Lobban F, Barrowclough C. An interpersonal CBT framework for involving relatives in interventions for psychosis: evidence base and clinical implications. Cognit Ther Res. 2016; 40(2):198-215.

10. Wright LM, Leahey M. Nurses and families: a guide to family assessment and intervention. 6th ed. Philadelphia, PA: F.A. Davis; 2013.

11. Onocko-Campos RO. Fale com eles! o trabalho interpretativo e a produção de consenso na pesquisa qualitativa em saúde: inovações a partir de desenhos participativos. Physis. 2011; 21(4):1269-86.

12. McFarlane WR. Family interventions for schizophrenia and the psychoses: a review. Fam Process. 2016; 55(3):460-82.

13. Lavis A, Lester H, Everard L, Freemantle N, Amos T, Fowler D, et al. Layers of listening: qualitative analysis of the impact of early intervention services for firstepisode psychosis on carers' experiences. Br J Psychiatry. 2015; 207(2):135-42.

14. Macleod S, Elliott L, Brown R. What support can community mental health nurses deliver to carers of people diagnosed with schizophrenia? Findings from a review of the literature. Int $\mathrm{J}$ Nurs Stud. 2011; 48:100-20.

15. Fiorati RC, Saeki T. As dificuldades na construção do modo de atenção psicossocial em serviços extra-hospitalares de saúde mental. Saúde Debate. 2013; 37(97):305-12.

16. Oliveira IC, Rocha J, Rocha RA, Lopes VA, Corrêa DP, Bellanda $\mathrm{PR}$, et al. As percepções do cuidado em saúde mental pelos profissionais de saúde de uma unidade básica de saúde do município de Itajaí-SC. Saúde Transform Soc. 2015; 6(2):44-53.

17. Dixon L, Mcfarlane WR, Lefley H, Lucksted A, Cohen M, Falloon I, et al. Evidence-based practices for services to families of people with psychiatric disabilities. Psychiatr Serv. 2001; 52(7):903-910.

18. Murphy N, Withnell N. Assessing the impact of delivering family interventions training modules: findings of a small-scale study. Ment Health Nurs. 2013; 33(5):1013.

19. Eassom E, Giacco D, Dirik A, Priebe S. Implementing family involvement in the treatment of patients with psychosis: a systematic review of facilitating and hindering factors. BMJ Open. 2014; 4(10): e006108.

20. Tacia L, Biskupski K, Pheley A, Lehto RH. Identifying barriers to evidence-based practice adoption: a focus group study. Clin Nurs Stud. 2015; 3(2):90-96.

RECEBIDO: $29 / 06 / 2018$

APROVADO: $29 / 11 / 2018$

PUBLICADO: $12 / 2018$ 